\title{
The complexities of bacterial-fungal interactions in the mammalian gastrointestinal tract
}

\author{
Eduardo Lopez-Medina ${ }^{1}$ and Andrew Y. Koh ${ }^{2, *}$ \\ ${ }^{1}$ Department of Pediatrics, Universidad del Valle and Centro de Estudios en Infectología Pediátrica, Cali, Colombia. \\ ${ }^{2}$ Departments of Pediatrics and Microbiology, University of Texas Southwestern Medical Center, Dallas, Texas, USA \\ * Corresponding Author: \\ Andrew Y. Koh, Department of Pediatrics and Microbiology, University of Texas Southwestern Medical Center, Dallas, Texas, USA; \\ Tel: +1 214 648-3896; Fax +1 214 648-3122; E-mail: Andrew.koh@utsouthwestern.edu
}

The mammalian gastrointestinal (GI) tract is host to trillions of microorganisms (including bacteria, fungi, and viruses), some of which can cause invasive and potentially life-threatening infections when the host's immune defenses are compromised. Cancer and stem cell transplant patients are particularly vulnerable to microorganisms translocating from the gut, with gram-negative bacteria such as Pseudomonas aeruginosa and the most common human fungal pathogen Candida albicans having significant medical impact. While much has been learned by studying fungal or bacterial pathogens in isolation, the interactions of bacteria and fungi living within the mammalian host are not well understood and may have significant consequences in the pathogenesis of infectious diseases. Here, we showcase two studies that explore bacterial-fungal interactions that modulate infectious disease pathogenesis: one in which commensal bacteria mediate host suppression of $C$. albicans gut colonization; the other in which $C$. albicans mediates suppression of $P$. aeruginosa virulence in the gut. Understanding how microbes interact and antagonize each other in specific body niches may help us identify new potential therapeutic targets for preventing or treating microbial infections.

With advances in medicine, particularly in cancer therapy and transplantation medicine, a burgeoning population of immunocompromised patients has emerged. These patients are highly vulnerable to infections with opportunistic pathogens. As a result, the use of prophylactic or empiric antibiotics to prevent bacterial, fungal, and viral infections is the standard of care. As a consequence of increased antibiotic exposure, the development of microbial antibiotic resistance is rising, and the unintended deleterious effect of antibiotics on resident microbiota is starting to be appreciated.

An imbalance in gut microbiota populations, also known as dysbiosis, can be precipitated by host genetics, dietary changes, and most notably exposure to antibiotics. There is a growing body of evidence that gut dysbiosis can be associated with a number of human pathogenic states. For instance, in cancer and stem cell transplant patients, endogenous bacteria and fungi can translocate from the gut and cause life-threatening bloodstream infections. Thus, when gram-negative bacteria (e.g. Enterobacteriaceae such as E. coli or Enterococcal spp.) burden increases in the gut, the risk of invasive bacteremia with these pathogens significantly increases [1].

Commensal anaerobic bacteria in the gut provide a key defense mechanism by inhibiting the growth of potentially pathogenic bacteria. One possible mechanism for maintaining pathogen $\mathrm{Gl}$ colonization resistance involves commensal anaerobe induction of mucosal immune effectors (e.g. antimicrobial peptides, AMP) that kill the pathogen. Manipulation of these mucosal immune effectors can not only decrease GI colonization with potentially pathogenic bacteria but also decrease the risk of invasive infection [2].

Gut commensal anaerobic bacteria can also modulate commensal fungi gut colonization $[3,4]$. Commensal fungi, notably Candida spp, colonize the GI tracts of numerous mammals, including humans. In hosts with a competent immune system, colonization with Candida spp. does not result in disease or infection. But severely immunocompromised patients (cancer, transplant, neonatal and inten-

Comment on Fan, D., Coughlin, L.A., Neubauer, M.M., Kim, J., Kim, M.S., Zhan, X., Simms-Waldrip, T.R., Xie, Y., Hooper, L.V., and Koh, A.Y. (2015). Activation of HIF-1alpha and LL-37 by commensal bacteria inhibits Candida albicans colonization. Nat Med 21(7):808-14. and LopezMedina, E., Fan, D., Coughlin, L.A., Ho, E.X., Lamont, I.L., Reimmann, C., Hooper, L.V., and Koh, A.Y. (2015). Candida albicans Inhibits Pseudomonas aeruginosa Virulence through Suppression of Pyochelin and Pyoverdine Biosynthesis. PLoS Pathog 11(8): e1005129. 
sive care unit patients) are at high risk for developing invasive Candida infection, many of which are thought to originate from the gut.

Since colonization is a prerequisite for both bacterial and fungal invasive disease, studying the interactions of gut bacterial and fungi and understanding how these interactions affect not only colonization but invasive infectious disease pathogenesis will be critical for developing novel methods to prevent these infections. With this thought in mind, our research group focused on the long-standing observation that adult mice are resistant to $\mathrm{Gl}$ colonization by $C$. albicans (CA), the most common human fungal pathogen. Germ-free mice and neonatal mice (with immature gut microbiota), however, can be sustainably colonized with CA. Furthermore, CA colonization resistance in mice is abrogated by antibiotic treatment, but only antibiotics most effective in depleting (killing) anaerobic bacteria resulted in the highest CA colonization levels. These data suggest that a mature and intact gut microbiota, particularly commensal anaerobes, are critical for maintaining CA resistance in mice [3].

Gut microbiota profiling (using both 16S rRNA sequencing and bacterial group specific QPCR) of CA colonization-resistant (no antibiotic treatment or antibiotics that did not deplete anaerobes) or CA colonization-susceptible (treated with anti-anaerobic antibiotics) revealed that Bacteroidetes and clostridial Firmicutes were the most effec- tive in promoting CA colonization resistance. Not surprisingly, endogenous gut fungi levels also increase in mice treated with anti-anaerobic antibiotics. Antibiotic exposure in humans has long been associated with increased risk of superficial fungal or yeast infections (i.e. oral thrush, yeast vaginitis, or yeast diaper rash). When antibiotic-treated or germ-free CA-colonized mice are orally gavaged with a representative Bacteroidetes (Bacteroides thetaiotamicron) or clostridial Firmicute (Blautia producta), CA is eliminated from the GI tract, whereas other representatives of the gut microbiota, including other commensal anaerobes, fail to promote CA gut colonization resistance. What is most striking, however, is that two Bacteroidetes species belonging to the same genus could have divergent effects on CA colonization: $B$. theta promoting CA reduction while Bacteroides fragilis having no appreciable affect on CA colonization [3]. Thus, microbial species-specific effects can drive distinct host responses and/or result in unique microbiota phenotypes [5].

The mechanistic basis for commensal anaerobic induction of CA resistance appears to rely on the induction of gut-derived host immune effectors, similar to how commensal anaerobes maintain Enterobacteriaceae or Enterococcal colonization resistance (Figure $1 \mathrm{~A})$. Both $B$. theta and $B$. producta induce greater colonic expression of the transcription factor HIF-1a, an important regulator of mammalian innate immunity, and the cathelicidin antimi-
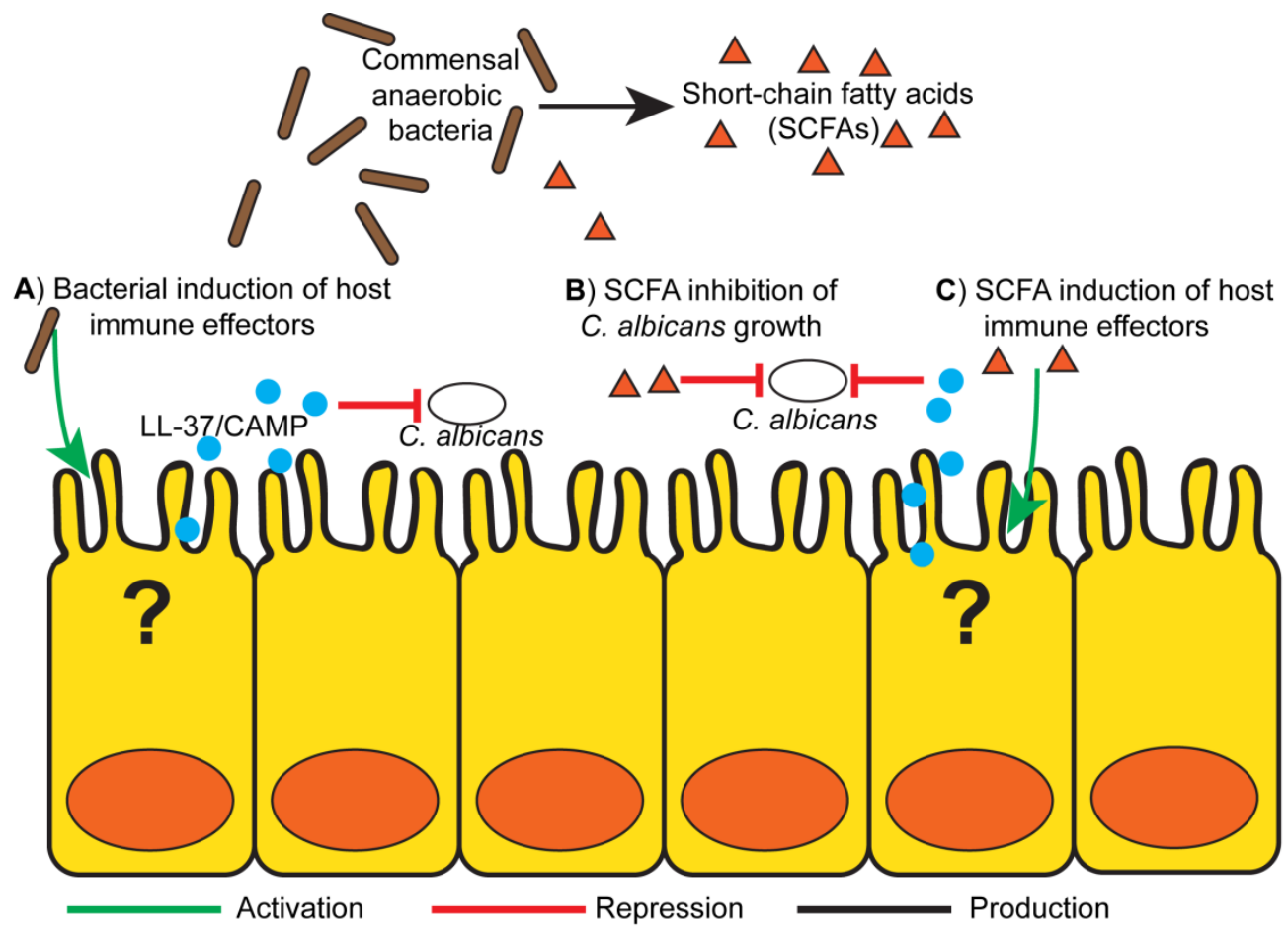

Figure 1: Proposed mechanism of gut bacteria mediated Candida albicans gastrointestinal colonization resistance. (A) Commensal gut bacteria, particularly obligate anaerobes (i.e. Bacteriodetes thetaiotamicron, Blautia producta), induce production of colonic host immune effectors, specifically antimicrobial peptides such as LL-37/CAMP which have Candicidal activity. (B) Bacterially derived fermentation products, short-chain fatty acids (SCFAs) may have a direct effect on C. albicans growth and colonization. (C) SCFAs can induce production of colonic antimicrobial peptides, including cathelicidins (LL-37/CAMP) and defensins that have activity against C. albicans. 
crobial peptide LL-37 (CAMP, the mouse ortholog) with anti-Candida activity, as compared with other commensal bacteria. In addition to microbial induced indirect host effects, both $B$. theta and $B$. producta produce small-chain fatty acids (SCFAs) that can induce host immune responses, including induction of Treg cells [6] and AMPs [7] (Figure 1C). Furthermore, SCFAs at physiologically relevant doses directly inhibits CA growth in vitro and decreases CA colonization in mice, suggesting that bacterially produced SCFAs may play a critical role in CA resistance [3] (Figure 1B).

Pharmacologic activation of HIF-1A using the Koa hoale seed derivative L-mimosine induces LL-37 production and ultimately reduces CA levels in an in vitro fungicidal assay using co-cultured human colonocytes, and this effect is nullified by HIF1A knockdown. When applying a similar strategy in vivo, CA GI colonization levels significantly decreased in wild-type mice treated with mimosine but not in mimosine-treated mice with Hif1a deleted from the intestinal epithelium. Strikingly, the mimosine-induced CA colonization reduction translated to a $50 \%$ decrease in mortality from CA invasive disease. Similar approaches of HIF1a activation of myeloid cells $[8,9]$ and keratinocytes [10] have been used to combat acute bacterial infection. Using Camp knockout mice, CAMP was also necessary for the mimosine-induced antifungal effects [3].

While HIF- $1 \alpha$ and CAMP are required for the commensal anaerobe-induced production against CA colonization in antibiotic treated mice, this was not true in the absence of antibiotics. It may be that with an intact and mature gut microbiota, the loss of one immune pathway (e.g. HIF/CAMP) is easily compensated for by other redundant immune effectors (e.g. other AMPs such as defensins) that also may maintain CA colonization resistance. In the setting of a markedly stressed and imbalanced gut microbiota (e.g. after antibiotics), the gut microbiota (input) stimulus may be so diminished that the loss of one immune pathway cannot be compensated for.

While mice are CA colonization resistant, reportedly up to $80 \%$ of human are colonized with CA [11]. How is this discrepancy explained? Estimates of high CA human colonization rates were based on studies conducted in western societies. More recent studies of humans living in remote and "traditional or primitive" societies exhibit a CA GI carriage rate of less than $10 \%[12,13]$, whereas other Candida spp. are more prevalent, consistent with the mycobiota of other mammals including rodents. Therefore, CA may not be an expected or normal member of the commensal gut microbiota in humans, but perhaps a more recently acquired "commensal" resulting from advances in human technology (i.e. treatment antibiotics, antibiotics in the food chain, and adoption of diets with greater refined carbohydrate and fat content) that induce gut microbiota changes which promote CA colonization. We have recently identified a human CA stool strain that is able to colonize the GI tract of mice with intact gut microbiota, and this clinical isolate is genetically distinct from the CA strains we used in the study described above (unpublished observation, AYK).

In summary, anaerobic commensal bacteria, particularly Bacteroidetes and clostridial Firmicutes clusters IV and XIVa, prevent or reduce GI C. albicans colonization in the murine model through activation of HIF1A and LL-37. In these severely immunocompromised cancer or stem cell transplant patients, augmenting innate cellular function or mucosal integrity is virtually impossible, so maintaining an intact gut microbiota and thus boosting Gl mucosal immune defenses to reduce $\mathrm{Gl}$ colonization of potentially pathogenic pathogens (such as CA) may be a novel method for preventing infections in these high-risk patients.

While the previous study [3] highlighted the importance of gut commensal bacteria inhibiting gut fungi, the corollary (fungi inhibiting or antagonizing a bacterium) is obviously possible. Therefore, we also chose to study the interactions of CA and the bacterium $P$. aeruginosa (PA): two pathogens that often inhabit the same body niches (gut, lung, burn wounds) and can cause devastating "opportunistic" infections in the immunocompromised host. The gut was used as the body niche of interest, since PA has a long and storied past of causing invasive bloodstream infections in cancer patients and thought to originate from the gut [14]. Despite being ubiquitous in the environment, PA is not a normal member of the human gut microbiota. Gut microbiota homeostasis (and thus pathogen colonization resistance) is a primary defense mechanism to prevent bacterial translocation from the gut. So in order to overcome the gut's inherent resistance to PA colonization, antibiotics are utilized to deplete commensal anaerobes in the mouse gut and thus promote PA (and CA) gut colonization [15].

Interestingly, while CA does not affect PA's ability to colonize the murine $\mathrm{GI}$ tract, the presence of CA prevents PA dissemination in the setting of neutropenia. Intact cellular immunity, particularly neutrophils, is another key defense mechanism against bacterial translocation from the gut and is sufficient for PA dissemination (but not for CA dissemination) $[4,15]$. CA inhibition of PA dissemination is not strain dependent and holds true when testing three PA and four CA strains (in all combinations) [16].

In vivo PA transcriptome analysis (using RNA-Seq) reveals CA-induced suppression of PA pyochelin and pyoverdine biosynthetic pathways. Pyochelin and pyoverdine play a critical role in iron acquisition and virulence. So one possible explanation as to why CA would suppress PA pyochelin and pyoverdine expression is to provide a competitive advantage for iron. Increasing iron concentrations can also inhibit PA pyochelin and pyoverdine gene expression, but the presence of CA does not increase overall iron levels in the mouse gut. Given the suppression of iron acquisition via pyochelin/pyoverdine, PA could be expected to have difficulty growing and thus lower GI colonization levels. But deletion of pyochelin and pyoverdine has no effect on PA GI colonization. As a testament to the importance of iron, PA (and other microorganisms) utilizes additional strategies to acquire iron (e.g. FeoABC system in 
A

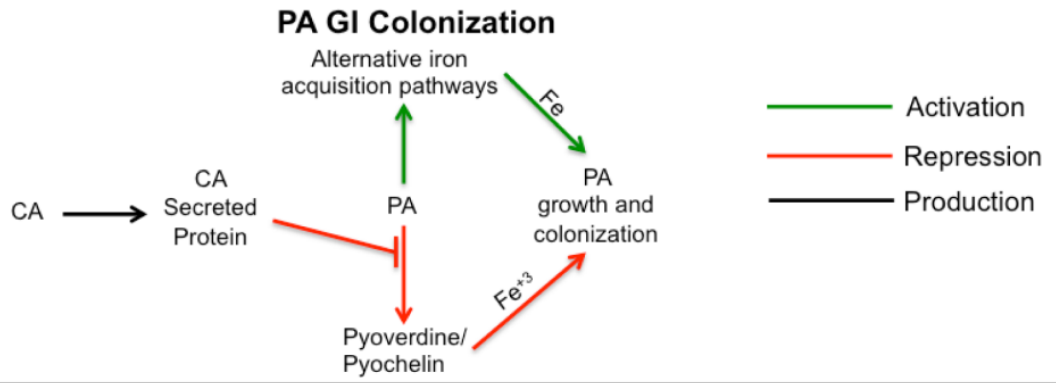

B

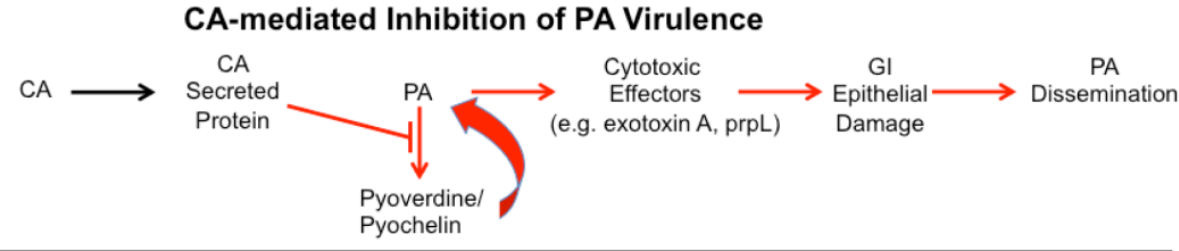

C

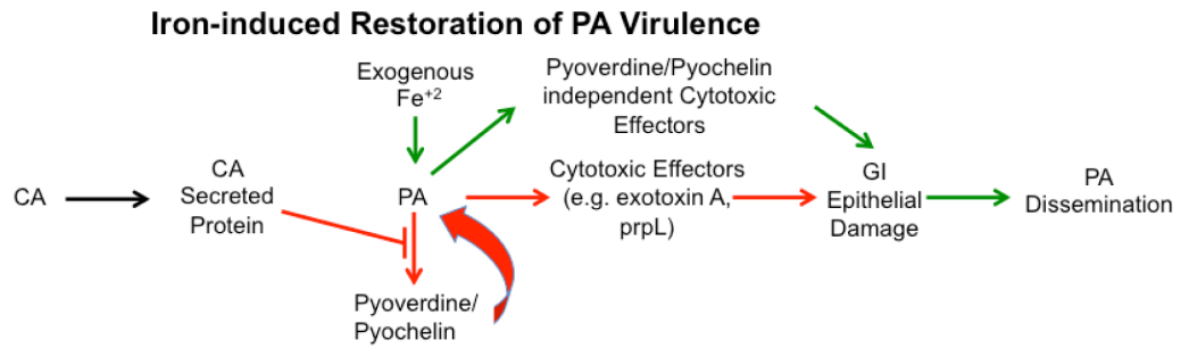

Figure 2. Proposed mechanism of Candida albicans (CA) inhibition of Pseudomonas aeruginosa (PA) virulence in the gut. (A) PA gastrointestinal (GI) colonization. Despite inhibition of pyoverdine and pyochelin gene expression by CA, PA is able to colonize the murine $\mathrm{GI}$ tract, perhaps by utilizing alternative iron acquisition pathways (e.g. FeoABC system) that allow sustained growth and colonization of the gut. (B) CAmediated Inhibition of PA Virulence. CA inhibits PA pyochelin and pyoverdine expression, most likely through a secreted protein. Production of PA extracellular virulence effectors, such as PrpL and exotoxin A, are decreased. Host gut epithelial integrity remains intact,

and PA dissemination is prevented. (C) Iron-induced restoration of PA virulence. Iron supplementation in PA/CA co-colonized mice induces pyochelin-pyoverdine independent PA cytotoxic effector molecular production leading to increased gut permeability and mucosal barrier damage. PA can now disseminate from the gut.

PA), thus may allow sustained growth and colonization of the GI tract (Figure 2A).

Deleting pyochelin or pyoverdine genes, however, significantly decreases the mortality from $P$. aeruginosa dissemination. Interestingly, pyoverdine is not only a siderophore important for iron acquisition but also a signaling molecule that induces the production of two extracellular virulence factors, the protease PrpL and exotoxin A [17]. Since an intact intestinal mucosal barrier is another primary defense mechanism preventing microbial translocation from the GI tract in humans and mice $[4,15,18]$, we postulated that CA-mediated protection from PA invasive infection might be due to inhibition of PA extracellular cytotoxic molecule production (i.e. exotoxin A, PrpL, etc). Indeed, CA (more specifically $C A$-secreted proteins) inhibits the production of PA extracellular cytotoxic molecules, such as exotoxin $A$, and thus inhibits the cytotoxic effect on cultured colonocytes [16]. These findings are consistent with our previous work demonstrating that disruption of PA cytotoxic virulence effector genes (e.g. Type III secretion system ExoU) also results in loss of cytotoxicity and significantly reduces virulence in a PA gut model without affecting PA GI colonization [19] (Figure 2B).

Moreover, CA-secreted factors (most likely extracellular proteins) inhibit PA pyochelin/pyoverdine gene expression and pyoverdine production in vitro. Interestingly, ad- ministration of heat-killed CA is unable to suppress PA virulence in mice, but CA culture supernatants and supernatant proteins significantly decrease PA virulence [16].

Finally, iron and iron-overload states have been shown to increase the virulence of bacteria [20], and consistent with these observations, iron supplementation restores PA virulence in PA-CA co-colonized mice. Iron supplementation does not promote PA expansion (increased burden) in the gut and likely has a negligible inhibitory effect on host phagocytosis (since the mice are profoundly neutropenic already). In fact, iron supplementation restores PA virulence by modulating gut epithelial integrity (increasing gut permeability) indirectly through PA and not directly via the host (Figure 2C). Hence, in contrast to the first study in which CA colonization modulation via host immune effectors ultimately results in decreased virulence, we describe a novel observation of a fungal-inhibition of bacterial effectors critical for virulence but not important for colonization.

In both studies by Fan, et al [3] and Lopez-Medina, et al [16], we utilize an "artificial" but now all too common niche, the antibiotic-treated gut with concomitant pathogenic microbe (PA or CA) expansion, to gain insight in bacterial-fungal interactions in clinically common scenarios: immunocompromised (neutropenia $\neg$ with or without gut epithelial disruption) hosts with antibiotic induced gut dysbiosis. Specifically, we describe two examples where 
bacterial-fungal interactions results in attenuation of invasive infectious disease: 1) commensal bacteria-mediated host suppression of CA colonization and 2) CA-mediated suppression of PA virulence. Thus, further studies of the synergistic or antagonistic bacterial-fungal interactions in the Gl tract may allow us to identify novel therapeutic methods (i.e CA-secreted proteins or gastrointestinal mucosal immune effectors) to prevent or control difficult to treat infections, an increasingly important clinical challenge given the rising tide of bacterial and fungal antibiotic resistance.

\section{ACKNOWLEDGMENTS}

This work was supported by the Roberta I. and Norman L. Pollock Fund (AYK) and Young Investigator Grant for Probiotics (AYK).

\section{REFERENCES}

1. Taur, Y., Xavier, J.B., Lipuma, L., Ubeda, C., Goldberg, J., Gobourne, A., Lee, Y.J., Dubin, K.A., Socci, N.D., Viale, A., Perales, M.A., Jenq, R.R., van den Brink, M.R., and Pamer, E.G. (2012). Intestinal domination and the risk of bacteremia in patients undergoing allogeneic hematopoietic stem cell transplantation. Clin Infect Dis 55(7): 905-914.

2. Brandl, K., Plitas, G., Mihu, C.N., Ubeda, C., Jia, T., Fleisher, M., Schnabl, B., DeMatteo, R.P., and Pamer, E.G. (2008). Vancomycin-resistant enterococci exploit antibiotic-induced innate immune deficits. Nature 455(7214): 804-807.

3. Fan, D., Coughlin, L.A., Neubauer, M.M., Kim, J., Kim, M.S., Zhan, X., Simms-Waldrip, T.R., Xie, Y., Hooper, L.V., and Koh, A.Y. (2015). Activation of HIF-1alpha and LL-37 by commensal bacteria inhibits Candida albicans colonization. Nat Med 21(7):808-14.

4. Koh, A.Y., Kohler, J.R., Coggshall, K.T., Van Rooijen, N., and Pier, G.B. (2008). Mucosal damage and neutropenia are required for Candida albicans dissemination. PLoS Pathog 4(2): e35.

5. Buffie, C.G., Bucci, V., Stein, R.R., McKenney, P.T., Ling, L., Gobourne, A., No, D., Liu, H., Kinnebrew, M., Viale, A., Littmann, E., van den Brink, M.R., Jenq, R.R., Taur, Y., Sander, C., Cross, J.R., Toussaint, N.C., Xavier, J.B., and Pamer, E.G. (2015). Precision microbiome reconstitution restores bile acid mediated resistance to Clostridium difficile. Nature 517(7533): 205-208.

6. Smith, P.M., Howitt, M.R., Panikov, N., Michaud, M., Gallini, C.A., Bohlooly, Y.M., Glickman, J.N., and Garrett, W.S. (2013). The microbial metabolites, short-chain fatty acids, regulate colonic Treg cell homeostasis. Science 341(6145): 569-573.

7. Schauber, J., Svanholm, C., Termen, S., Iffland, K., Menzel, T., Scheppach, W., Melcher, R., Agerberth, B., Luhrs, H., and Gudmundsson, G.H. (2003). Expression of the cathelicidin LL-37 is modulated by short chain fatty acids in colonocytes: relevance of signalling pathways. Gut 52(5): 735-741.

8. Peyssonnaux, C., Datta, V., Cramer, T., Doedens, A., Theodorakis, E.A., Gallo, R.L., Hurtado-Ziola, N., Nizet, V., and Johnson, R.S. (2005). HIF1alpha expression regulates the bactericidal capacity of phagocytes. The Journal of clinical investigation 115(7): 1806-1815.

9. Zinkernagel, A.S., Peyssonnaux, C., Johnson, R.S., and Nizet, V. (2008) Pharmacologic augmentation of hypoxia-inducible factor-1alpha with mimosine boosts the bactericidal capacity of phagocytes. J Infect Dis 197(2): 214-217.

10. Nizet, V., Ohtake, T., Lauth, X., Trowbridge, J., Rudisill, J., Dorschner, R.A., Pestonjamasp, V., Piraino, J., Huttner, K., and Gallo, R.L. (2001)

\section{CONFLICT OF INTEREST}

The authors declare no conflict of interest.

\section{COPYRIGHT}

(C) 2016 Lopez-Medina and Koh. This is an open-access article released under the terms of the Creative Commons Attribution (CC BY) license, which allows the unrestricted use, distribution, and reproduction in any medium, provided the original author and source are acknowledged.

Please cite this article as: Eduardo Lopez-Medina and Andrew Y. Koh (2016). The complexities of bacterial-fungal interactions in the mammalian gastrointestinal tract. Microbial Cell 3(5): 191195.

Innate antimicrobial peptide protects the skin from invasive bacterial infection. Nature 414(6862): 454-457.

11. Bougnoux, M.E., Diogo, D., Francois, N., Sendid, B., Veirmeire, S., Colombel, J.F., Bouchier, C., Van Kruiningen, H., d'Enfert, C., and Poulain, D. (2006). Multilocus sequence typing reveals intrafamilial transmission and microevolutions of Candida albicans isolates from the human digestive tract. Journal of clinical microbiology 44(5): 1810-1820.

12. Angebault, C., Djossou, F., Abelanet, S., Permal, E., Ben Soltana, M., Diancourt, L., Bouchier, C., Woerther, P.L., Catzeflis, F., Andremont, A., d'Enfert, C., and Bougnoux, M.E. (2013). Candida albicans is not always the preferential yeast colonizing humans: a study in Wayampi Amerindians. J Infect Dis 208(10): 1705-1716.

13. Xu, J., and Mitchell, T.G. (2003). Geographical differences in human oral yeast flora. Clin Infect Dis 36(2): 221-224.

14. Tancrede, C.H., and Andremont, A.O. (1985). Bacterial translocation and gram-negative bacteremia in patients with hematological malignancies. J Infect Dis 152(1): 99-103.

15. Koh, A.Y., Priebe, G.P., and Pier, G.B. (2005). Virulence of Pseudomonas aeruginosa in a murine model of gastrointestinal colonization and dissemination in neutropenia. Infect Immun 73(4): 2262-2272.

16. Lopez-Medina, E., Fan, D., Coughlin, L.A., Ho, E.X., Lamont, I.L., Reimmann, C., Hooper, L.V., and Koh, A.Y. (2015). Candida albicans Inhibits Pseudomonas aeruginosa Virulence through Suppression of Pyochelin and Pyoverdine Biosynthesis. PLoS Pathog 11(8): e1005129.

17. Lamont, I.L., Beare, P.A., Ochsner, U., Vasil, A.I., and Vasil, M.L. (2002). Siderophore-mediated signaling regulates virulence factor production in Pseudomonasaeruginosa. Proc Natl Acad Sci U S A 99(10): 7072-7077.

18. Berg, R.D. (1999). Bacterial translocation from the gastrointestinal tract. Adv Exp Med Biol 473: 11-30.

19. Koh, A.Y., Mikkelsen, P.J., Smith, R.S., Coggshall, K.T., Kamei, A., Givskov, M., Lory, S., and Pier, G.B. (2010). Utility of in vivo transcription profiling for identifying Pseudomonas aeruginosa genes needed for gastrointestinal colonization and dissemination. PLoS One 5(12): e15131.

20. Litwin, C.M., and Calderwood, S.B. (1993). Role of iron in regulation of virulence genes. Clinical microbiology reviews 6(2): 137-149. 